\title{
DOENÇA DO DISCO INTERVERTEBRAL EM CÃES DA RAÇA DACHSHUND: UMA REVISÃO DE LITERATURA
}

\author{
Belissa Ferreira Cecim ${ }^{\star}$
}

\begin{abstract}
RESUMO: Este trabalho tem como objetivo apresentar uma revisão de literatura sobre a doença do disco intervertebral em cães da raça Dachshund, condição frequente na clínica de pequenos animais, em que os discos intervertebrais podem sofrer degeneração ou ruptura, que resultam na contusão e/ou compressão da medula espinhal. Existem duas apresentações clássicas da doença, a Hansen tipo I: extrusão de disco de acometimento clínico agudo e Hansen tipo II: protrusão de disco de progressão clínica lenta do disco intervertebral. Identificouse recentemente a extrusão aguda não compressiva do núcleo pulposo e a extrusão aguda do núcleo pulposo hidratado. Os sinais clínicos incluem: hiperestesia à palpação da coluna, ataxia sensorial, déficits proprioceptivos, alteração de resposta dos reflexos espinhais, paresia ambulatória ou não ambulatória. 0 diagnóstico baseia-se no histórico, anamnese, exame clínico neurológico e exames de imagem complementares, tais como mielografia, ressonância magnética ou tomografia computadorizada. 0 tratamento clínico é constituído de repouso absoluto, uso de analgésicos e anti-inflamatórios; já o tratamento cirúrgico é indicado quando há alto grau de disfunção neurológica, visando à descompressão da medula espinhal. A enfermidade pode acometer cães de todas as raças e idades, porém as raças condrodistróficas, como os Dachshund, têm de dez a 12 vezes mais risco de desenvolver a doença do que as demais raças e, até $24 \%$ deles apresentam a patologia durante a vida. Esta prevalência é superior às outras raças, 0 que é explicado tanto por sua conformação anatômica como pela predisposição genética específica da raça, com genes de suscetibilidade já identificados nos cromossomos 12 e 18.
\end{abstract}

PALAVRAS-CHAVE: Mielopatia; Discopatia; Raças condrodistróficas; Teckel.

\section{DISEASE OF THE INTERVERTEBRAL DISC IN DACHSHUNDS: REVIEW OF THE LITERATURE}

\begin{abstract}
The literature on diseases of the intervertebral disc in Dachshund dogs is analyzed. It is a frequent clinical condition in small animals by which intervertebral discs degenerate or rupture, with contusion and/ compression of the spine medulla. There are two classical modes: Hansen Type I, or the extrusion of the disc with acute clinical effects, and Hansen Type II, with protrusion of the disc with a slow clinical progression of the intervertebral disc. The non-compressive acute extrusion of the pulpous nucleus and the acute extrusion of the hydrated pulpous nucleus have been recently been identified. Clinical diagnosis includes hyperaesthesia on the touching of the spine, sensorial ataxia, proprioceptive deficits, response changes in spinal reflexes, ambulatory or non-ambulatory paresis. Diagnosis is based on records, neurological clinical exams and complementary image exams such as myelography, magnetic resonance or computerized tomography. Clinical treatment consists of absolute rest, analgesics and anti-inflammatory medicine. Surgery is recommended when there is high neurological dysfunction degree to ease decompression of the spinal medulla. Disease may affect dogs of all types and ages, although condrodystrophic races, such as the Dachshund, may have between 10 and 12 times the chance of developing the disease. Prevalence is higher than in other races and may be due to anatomic conformation and race-specific genetic predisposition, with susceptibility genes in chromosomes 12 and 18 .
\end{abstract}

KEYWORDS: Myelopathy; Discopathy; Condrodystrophic races; Teckel.

\footnotetext{
Medicina Veterinária pelo Centro Universitário Ritter dos Reis, UniRITTER. Especialização em andamento em Neurologia Veterinária de pequenos animais pela Faculdade Qualittas, Campinas, SP, Brasil. E-mail: cecim.belissa@gmail.com
} 


\section{INTRODUÇÃO}

A doença do disco intervertebral (DDIV) possui uma ocorrência frequente na clínica de pequenos animais (BRISSON, 2010). Nesta afecção, os discos intervertebrais (DIV) que possuem uma camada externa fibrosa - denominada anel fibroso (AF) - e uma camada interna gelatinosa - denominada núcleo pulposo (NP) - podem sofrer degeneração ou lesão e, consequentemente, ocasionar compressão da medula espinhal (ME) (PACKER et al., 2016).

Existem duas apresentações clássicas da doença, a Hansen tipo I ou extrusão de DIV em que a velocidade de extrusão e a quantidade de material extruído estão diretamente ligadas à gravidade da manifestação da doença; e a denominada Hansen tipo II ou protrusão de DIV, que envolve o espessamento progressivo do AF dorsal, o qual protrui dorsalmente para 0 interior do canal vertebral. Identificou-se recentemente que extrusões agudas podem ocorrer em pacientes que tenham NP não degenerado ou minimamente degenerado: a extrusão aguda não compressiva do NP (EANCNP) e a extrusão aguda do NP hidratado (EANPH). É importante o diagnóstico correto pelo exame de imagem complementar, como a ressonância magnética, para diferenciar uma extrusão da outra e auxiliar na tomada de decisão para 0 tratamento adequado (BRISSON, 2010; BERGKNUT et al., 2013; DE DECKER; FENN, 2018). As extrusões de disco ocorrem normalmente em raças condrodistróficas e as protrusões de disco ocorrem mais em raças não condrodistróficas (DA COSTA; DEWEY, 2017). Dentre as raças condrodistróficas, os cães da raça Dachshund têm de dez a 12 vezes maior risco de desenvolver a doença do que as demais raças e, cerca de 19-24\% deles, apresentam sinais de DDIV durante a vida (PACKER et al., 2016).

A dor é proeminente na maioria dos cães afetados pela doença, pela compressão que o material herniado pode e/ou deve provocar sobre as meninges e, ocasionalmente, sobre as raízes nervosas. Os sinais clínicos dependem do local da lesão, gravidade da contusão e do grau de compressão da medula espinhal, podendo aparecer de modo agudo ou não
(DENNY; BUTTERWORTH, 2006). Os pacientes, além da dor, podem ainda demonstrar sinais clínicos como ataxia com déficits proprioceptivos e disfunções motora e sensorial (PACKER et al., 2016).

0 diagnóstico baseia-se no histórico, exame clínico neurológico e exames de imagem complementares (mielografia, ressonância magnética ou tomografia computadorizada). Para confirmação do diagnóstico é necessário realizar exames de mielografia, tomografia computadorizada (TC) ou ressonância magnética (RM) (DA COSTA; DEWEY, 2017).

0 tratamento para a DDIV pode ser clínico ou cirúrgico, e a escolha do protocolo deverá ser de acordo com a gravidade da disfunção neurológica. 0 tratamento clínico é constituído de repouso absoluto em gaiolas, com restrição de movimento, por quatro a seis semanas, associado ao uso de analgésicos e anti-inflamatórios (FESTUGATTO et al., 2008). 0 tratamento cirúrgico é indicado no caso de insucesso do tratamento clínico e visa a descompressão da medula espinhal, sendo que as técnicas variam de acordo com o local da lesão: laminectomia dorsal, hemilaminectomia, pediculectomia ou corpectomia (DA COSTA; DEWEY, 2017).

0 prognóstico para a DDIV vai depender da localização, sinais neurológicos e clínicos apresentados e tratamento realizado (BRISSON, 2010). Em cães que apresentam dor profunda, após cirurgia descompressiva, são relatadas altas taxas de recuperação, de 86-96\% (BUSH et al., 2007) comparando com a taxa de recuperação de $50 \%$ dos cães que perderam a dor profunda, se operados em até 24 horas pós-trauma (AlKAWA et al., 2012).

A literatura demonstra que pode ocorrer recidivas pelo mesmo DIV ou por acometimento em outros locais. Quando ocorrer uma recidiva, os sinais clínicos tendem a ser de maior gravidade, devendose avaliar o animal novamente e dependendo do seu grau de disfunção (grau I - apenas hiperestesia; grau II - ataxia e paresia ambulatória; grau III - paresia não ambulatória; grau IV - plegia com nocicepção preservada; grau $\mathrm{V}$ - plegia com perda de nocicepção), 
decidir pelo melhor protocolo para cada paciente, se pelo protocolo clínico (analgésicos, anti-inflamatórios, repouso absoluto, fisioterapia) ou, quando os sinais clínicos são mais graves, pelo protocolo cirúrgico (ARIAS et al., 2007).

\section{REVISÃO DA LITERATURA}

\subsection{ANATOMIA DA COLUNA VERTEBRAL}

A coluna vertebral do cão é composta por sete vértebras cervicais, 13 vértebras torácicas, sete vértebras lombares, três vértebras fundidas sacrais e vértebras coccígeas que variam em número de acordo com a raça do animal. A vértebra divide-se em corpo, arco vertebral e seus processos articulares, e sua conformação depende de sua localização. Com exceção da articulação atlantoaxial e das vértebras sacrais, existe um DIV no espaço entre dois corpos vertebrais adjacentes (DA COSTA; DEWEY, 2017).

0 DIV tem como principal função conectar as vértebras, fornecer flexibilidade à coluna vertebral $\mathrm{e}$ absorver impactos. Composto basicamente por NP e AF (BRISSON, 2010), o NP tem forma oval, é translúcido e de aparência mucoide, presente no centro do DIV; é uma substância gelatinosa, composto por proteoglicanos, que apresentam grande quantidade de água, o que confere sua capacidade de amortecimento (FERNÁNDEZ; BERNARDINI, 2010). 0 NP é envolvido pelo AF, sendo este constituído por fibras lamelares múltiplas e concêntricas. 0 AF possui maior espessura ventralmente que 0 dorsalmente, motivo este que predispõe sua ruptura no aspecto dorsal (BERGKNUT et al., 2013).

Além do DIV, existem ligamentos que ajudam a conferir estabilidade à coluna vertebral. Dentre eles, estão 0 ligamento longitudinal dorsal, que recobre 0 aspecto dorsal dos corpos vertebrais, passando pelo assoalho do canal vertebral e sobre os AF do DIV, e 0 ligamento longitudinal ventral, que se estende pelo aspecto ventral dos corpos vertebrais de C2 até 0 sacro. Na região torácica, existe outro ligamento, o intercapital, que emerge da cabeça de uma costela, passa sob o ligamento longitudinal dorsal, e se insere na cabeça da costela oposta, estando ausente no $1^{\circ}$, $12^{\circ}$ e $13^{\circ}$ pares de costela. Desta forma, o ligamento intercapital diminui a incidência de discopatias nas regiões onde está presente (TOOMBS; WATERS, 2007).

A medula espinhal (ME) é composta de substância cinzenta no centro e branca em sua periferia. Encontra-se dentro do canal vertebral, estendendo-se da altura do forame magno até a altura da sexta vértebra lombar, podendo o cone espinhal estar disposto mais caudalmente de acordo com a raça do animal (ARIAS et al., 2007).

A ME é dividida em segmentos espinhais, delimitados pela identificação bilateral de um par de raízes dorsais (sensoriais) e um par de raízes ventrais (motoras), as quais vão chegar/deixar o canal vertebral pelos respectivos forames intervertebrais. Uma vez que na região cervical existem oito segmentos espinhais e apenas sete vértebras, o primeiro par de raiz espinhal passa através forame alar, presente na asa do atlas. Caudalmente à região cervical, o número de segmentos espinhais e vértebras é 0 mesmo na região torácica (13), lombar (07), sacral (03) e caudal (pelo menos 05 segmentos caudais e número de vértebras varia de acordo com a raça). Nessas regiões, cada par de raiz espinhal passa através do forame intervertebral caudal a vértebra de mesmo número (SHARP; WHEELER, 2005), com exceção da região lombar, onde as raízes nervosas de $L 5, L 6, L 7$ encontram-se na vértebra lombar 4 , já os segmentos sacrais S1, S2, S3 localizam-se na quinta vértebra lombar e os segmentos coccígeos na vértebra lombar 6 (NELSON; COUTO, 2006).

\subsection{ETIOLOGIA}

A degeneração do DIV é um processo natural associado à idade do animal, que pode ou não ocasionar a DDIV (BRISSON, 2010). Diversas alterações bioquímicas e biomecânicas levam à degeneração do DIV. Quando ocorre a deterioração da qualidade da matriz, resulta em um efeito cascata, levando à falha 
estrutural do AF e NP, em que a deterioração de um componente leva a de outro, o que pode ocasionar ruptura ou abaulamento do disco (BERGKNUT et al., 2013).

Os cães de raças condrodistróficas (Daschshund, Basset Hound, Lhasa Apso, Poodle Miniatura, Shih Tzu, Beagle, entre outras) podem apresentar alterações no NP desde os três meses de idade (SMOLDERS et al., 2013) e com até um ano $90 \%$ deles poderão apresentar mineralização parcial ou total em vários DIV, por isso a maior probabilidade de herniação do material degenerado do NP para 0 canal vertebral (MCKEE, 2000; SMOLDERS et al., 2013). De acordo com KRANENBURG et al. (2013), os condrodistróficos têm 11,3 vezes mais chance de desenvolver extrusão do que protrusão.

Alguns cães condrodistróficos possuem hemivértebras, indicando assim a pré-disposição ao DDIV devido a sua estrutura anatômica alterada e possivelmente comprometimento de sua funcionalidade, podendo causar alteração no espaço intervertebral e consequentemente a extrusão do DIV (PENHA et al., 2010).

\subsection{DACHSHUND}

A predisposição da raça Dachshund deve-se à sua conformação condrodistrófica "Ionga e baixa" e está associada a um retrógeno que codifica o fator de crescimento de fibroblastos 4 (FGF4), situado no cromossomo 18. Contudo, a DDIV apresenta uma etiologia multifatorial, envolvendo genes e fatores ambientais (PARKER et al., 2009).

Segundo Mogensen et al. (2012), a base hereditária de calcificações do DIV foi demonstrada no estudo genético em Dachshund no qual se verificou que 0 cromossomo 12 abrigou variações genéticas que afetaram 0 desenvolvimento da calcificação do DIV. Com base nestes resultados, a variação neste lócus da associação da doença parece constituir um fator determinante na calcificação do DIV. Segundo recente estudo de Stigen et al. (2019), com 25 Dachshunds, a histopatologia revelou calcificação em material de disco extrusado de todos os cães analisados.

Jensen e Christensen (2000) realizaram um estudo com oito famílias de Dachshund, em que demonstraram alta hereditariedade de 0,47 - 0,87 para calcificação de DIV baseado na relação paifilho. No mesmo estudo visualizou-se que se os pais tivessem calcificação em $91 \%$ dos DIV a prole também teria, enquanto se apenas um dos pais tivesse a calcificação do DIV, a presença na prole seria de 44\%, demostrando que a calcificação do DIV é hereditária. Corrobora Lappalalein et al. (2015) na sua pesquisa, indicando que os traços para herdabilidade são altos, sendo assim, o controle da reprodução seria possível selecionando apenas Dachshund com nenhuma ou poucas calcificações no DIV. De acordo com estudo realizado por Jensen et al. (2008), que comparou 0 número de calcificações com DDIV em Dachshund, na Dinamarca, quanto menos calcificações o animal tiver, menor seu risco para desenvolver DDIV.

Segundo estudo retrospectivo com Dachshunds de Dorn e Seath (2018), a castração aumenta o risco de DDIV nos machos castrados antes de um ano de idade e nas fêmeas quando castradas com qualquer idade. Considerando a prevalência da doença na raça, o risco da patologia associado à castração é um fator que deverá ser analisado individualmente pelo médico veterinário, vendo prós e contras para esta tomada de decisão.

\subsection{PATOFISIOLOGIA}

A DDIV é designada pela calcificação do DIV que leva a uma síndrome neurológica de contusão e/ ou compressão da ME e/ou raiz nervosa, consequência do deslocamento do DIV ou partes dele para o canal vertebral e forame intervertebral, respectivamente. A prevalência maior da DDIV é em cães não condrodistróficos a partir dos sete anos, e ambas as metaplasias podem ocorrer ao longo de toda coluna vertebral (BRISSON, 2010).

Mudanças degenerativas em raças condrodistróficas são referidas como metaplasia condroide -em que o NP sofre desidratação e é invadido 
por cartilagem hialina, ocasionando 0 enfraquecimento das fibras do AF 0 que pode resultar em perda das propriedades hidroelásticas e da capacidade de resistir à pressão. $0 \mathrm{NP}$ gelatinoso e transparente é modificado para uma cor amarela fibrocartilaginosa; - já nas raças não condrodistróficos são referidas como metaplasia fibroide - processo degenerativo que ocorre com a desidratação progressiva do NP, que é substituído por fibrocartilagem (BRISSON, 2010). Ocasionando 0 abaulamento do NP dentro do AF já fragilizado, ocorrendo protrusão do DIV (SMOLDERS et al., 2013). A prevalência maior é em cães não condrodistróficos a partir dos sete anos. Ambas as metaplasias podem ocorrer ao longo de toda coluna vertebral (DE LAHUNTA; GLASS, 2009; BRISSON, 2010).

A Hansen tipo I - caracterizada pela extrusão de DIV que afeta geralmente cães de pequeno porte e condrodistróficos, após os dois anos de idade do animal (DA COSTA; DEWEY, 2017) - é associada à metaplasia condroide, onde ocorre a migração de maneira aguda do material do NP - que perde propriedades hidroelásticas e capacidade de resistir à pressão - rompendo-0 e migrando para 0 canal vertebral (OLBY; JEFFERY, 2012). 0 material do disco que sofreu extrusão é irregular, frágil, de aspecto granular, com sua coloração que varia de brancoamarelo para cinza-amarelo, ou, se houver lesão de algum seio venoso, de coloração cinza-vermelho, se com sangue misturado (BRISSON, 2010). Segundo Da Costa e Dewey (2017), a Hansen tipo I usualmente gera sinais clínicos de rápida evolução (minutos/dias).

A Hansen tipo II - caracterizada pela protrusão de DIV afeta geralmente cães não condrodistróficos, a maioria de grande porte (DENNY; BUTTERWORTH, 2006) - é associada à metaplasia fibroide que se caracteriza pela ruptura parcial do $\mathrm{AF}$, ocasionando uma extensão focal do NP - e AF para 0 canal vertebral, com uma protuberância lisa e arredondada, que dificilmente fica aderida à dura-máter provocando compressão da ME (BRISSON, 2010), ocorrendo protrusão do DIV (SMOLDERS et al., 2013) em que se observa um déficit neurológico progressivo e lento (FOSSUM, 2005).

Recentemente, foram identificados outros dois tipos de extrusão: a aguda não-compressiva do NP (EANCNP), também conhecida como Hansen tipo III e a aguda do NP hidratado (EANPH) (DE DECKER; FENN, 2018). A EANCNP é uma extrusão súbita do NP não degenerado que causa lesão na ME sem realizar compressão medular. A doença tem início hiperagudo e sinais clínicos frequentemente graves; segundo Fenn et al. (2016), 90\% dos animais afetados apresentam sinais lateralizados e não progressivos após as 24 horas iniciais dos sintomas, que ocorrem após a realização de exercícios ou trauma (DE DECKER; FENN, 2018), em que pode ocorrer uma pequena ruptura no AF, e 0 NP será extruído dorsalmente ao canal vertebral com grande força, gerando contusão focal na ME (DE RISIO et al., 2009). Border Collies são mais susceptíveis à EANCNP, embora qualquer raça possa desenvolver esta patologia (FENN et al., 2016).

Diferentemente das Hansen tipo I I II, na EANPH o material extruído está íntegro, ou seja, hidratado, e ele será reabsorvido ou dissipado, não ocorrendo compressão após sua reabsorção (DE RISIO et al., 2009). A EANPH tem fisiopatogenia desconhecida, a possibilidade é que a extrusão ocorra por uma fissura no AF resultante de mudanças súbitas na pressão $\mathrm{e}$ biomecânica do DIV (DOLERA et al., 2015). De acordo com Royaux et al. (2016), a EANPH tem propensão a atingir a região cervical, e a apresentação clínica mais frequente é a tetraparesia e tetraplegia não ambulatória, podendo afetar raças condrodistróficas e não condrodistróficas. Os sinais clínicos normalmente apresentam-se de forma grave e simétrico e a predileção da doença é por animais mais velhos, com idade média de nove anos. (MANUNTA et al., 2015).

A contusão da ME acontece em qualquer tipo de DDIV, e causa uma lesão mecânica primária seguida de eventos bioquímicos no tecido horas após a lesão sofrida (OLBY, 2010). Contusão, cisalhamento, laceração, aceleração ou desaceleração são causas da lesão traumática primária associadas à ME (ROWLAND et al., 2008). Segundo Olby et al. (2016), o resultado é o rompimento físico das membranas celulares causando hemorragia e consequente isquemia $e$ lesão neuronal e glial generalizada. A compressão 
afeta a perfusão da medula espinhal pela limitação da suprimento e oclusão da drenagem venosa e causa dano direto à mielina e axônios. Na lesão secundária ocorre a expansão da destruição do tecido ocasionando hemorragia, inflamação, alteração na concentração de íons intracelulares e destruição microvascular (ROWLAND et al., 2008).

\subsection{SINAIS CLÍNICOS}

Os sinais clínicos são influenciados por diversos fatores, como o volume de material herniado, força e velocidade do impacto inicial e tempo de compressão (ARIAS et al., 2007). A relação entre 0 diâmetro espinhal e 0 diâmetro do canal vertebral também é um fator importante. A ME será menos afetada em locais que o disco extruído tenha mais espaço para se acomodar (FERNÁNDEZ; BERNARDINI, 2010).

Clinicamente, a medula espinhal é dividida em quatro conjuntos de segmentos espinhais, cada qual originando uma síndrome clínica específica. São eles: segmento cervical entre C1 e C5, intumescência cervicotorácica entre $\mathrm{C} 6$ e T2, segmento toracolombar entre T3 e L3, e intumescência lombossacra L4-S3 (FOSSUM, 2005).

No segmento cervical (C1-5) quando ocorre lesão, os sinais clínicos são de NMS - paresia ou paralisia, reflexos e tônus de normais a aumentados e leve atrofia muscular. No segmento cervicotorácico (C6-T2), região de NMI, os sinais nos membros torácicos são de NMI - paresia ou paralisia, reflexos e tônus diminuídos ou ausentes e grave atrofia muscular, já nos membros pélvicos o sinal é de NMS. No segmento toracolombar apenas os membros pélvicos apresentam alteração, com sinais de NMS, e no segmento da intumescência lombossacra apenas os membros pélvicos apresentam sinal de NMI (DA COSTA; DEWEY, 2017).

Na DDIV cervical o sinal clínico predominante é hiperestesia no pescoço, posição de cabeça baixa, fraqueza do membro torácico, hemiparesia ou tetraparesia (SHARP; WHEELER, 2005; BRISSON, 2010).
Geralmente, a síndrome cervical pode não apresentar déficits neurológicos, por conta do maior diâmetro do canal vertebral em relação aos outros segmentos, portanto para ter um déficit neurológico a lesão terá que ser severa na compressão da ME (BRISSON, 2010). Segundo Hakozaki et al. (2015), o segmento C2-3 é o mais acometido na síndrome cervical em cães condrodistróficos, como os Dachshunds.

Já na DDIV toracolombar, os sinais clínicos são hiperestesia espinhal, cifose, relutância em se movimentar, disfunção urinária, disfunção fecal, ataxia sensorial, paresia e até paraplegia (SHARP; WHEELER, 2005; DENNY; BUTTERWORTH, 2006; BRISSON, 2010).

Segundo estudo de Rohdin et al. (2010), o local de maior acometimento de DDIV em Dachshund é a região toracolombar. Os principais sinais clínicos foram hiperestesia e paraparesia, com diminuição ou ausência de reações posturais nos membros pélvicos. Os discos mais comumente afetados pelas extrusões de DIV foram T12-T13 (28\%), seguido por T13-L1 (23\%), T11-T12 (15\%) e L1-L2 (10\%).

Um sinal clínico frequente na DDIV é a ocorrência de disfunção urinária, que no caso de acometimento de lesão entre a ponte e L7 da ME mais comumente no segmento toracolombar (NMS), 0 animal não consegue esvaziar a bexiga por estar com 0 reflexo detrusor lesionado ou anulado pela lesão inicial da $\mathrm{ME}$, à palpação da bexiga, a mesma fica rígida 0 que torna bem difícil a compressão vesical. Já quando a lesão é no NMl - região sacral e caudal da região lombar - também pode ocorrer lesão ou não no reflexo detrusor, porém por tônus diminuído, a musculatura fica flácida, provocando perda de urina constante pelo paciente (DA COSTA; DEWEY, 2017).

Após o exame neurológico, deve ser realizada uma graduação dos déficits neurológicos: grau I - apenas hiperestesia; grau II - ataxia e paresia ambulatória; grau III -paresia não ambulatória; grau IV - plegia com nocicepção preservada; grau V - plegia com perda de nocicepção (BRISSON, 2010).

De acordo com estudo realizado por Kranenburg et al. (2013), a probabilidade de DDIV cervical como Hansen tipo I é 2,2 vezes mais que a 
tipo Il; na DDIV toracolombar é de 3,2 vezes mais na Hansen tipo I do que no tipo II; já na DDIV Iombossacra a Hansen tipo I tem 31,4 vezes menos chance do que a Hansen tipo II. Nos cães condrodistróficos, a chance da síndrome cervical e toracolombar é de 1,1 a 1,8 vezes, respectivamente, mais do que nos cães não condrodistróficos.

\subsection{DIAGNÓSTICO}

0 diagnóstico é baseado na combinação de avaliação de anamnese, exame físico, exame neurológico e exames complementares de radiografia simples, mielografia, TC ou RM (FOSSUM, 2005; BRISSON, 2010; DA COSTA; DEWEY, 2017).

Avaliam-se no exame neurológico: nível de consciência, comportamento, postura e marcha, avaliação de nervos cranianos, reações posturais, reflexos segmentares espinhais, hiperestesia e nocicepção. No exame neurológico existe a possibilidade de determinar aproximadamente 0 local da lesão, extensão e gravidade. A manipulação do animal com lesão medular aguda deve ser muito cautelosa, tendo em vista o risco de exacerbar a lesão (SHARP; WHEELER, 2005).

No RX simples a confirmação do diagnóstico da doença é muito difícil, porém, são compatíveis com DDIV tipo I, caso haja diminuição do espaço intervertebral ou material calcificado dentro do canal vertebral (FOSSUM, 2005; BRISSON, 2010). Segundo Jensen e Arnbjerg (2001), as degenerações do DIV já podem ser visualizadas pelo $\mathrm{RX}$ aos dois anos de idade do Dachshund.

Em países nórdicos (Dinamarca, Finlândia e Noruega), a triagem para visualização de calcificações de DIV em Dachshund tem sido amplamente recomendada, onde o protocolo inclui RX látero-lateral de toda coluna. A calcificação é classificada de acordo com o número de DIV afetados, e 0 animal é livre se não tiver calcificação, risco leve se tiver até duas calcificações, risco moderado até quatro calcificações e risco grave se tiver mais que cinco calcificações. Na Finlândia, este protocolo realizado na faixa etária de dois a quatro anos é utilizado por mais de uma década (LAPPALAINEN et al., 2015).

Para um diagnóstico definitivo, deve-se realizar exames de imagem como mielografia, TC ou RM para permitir a localização da lesão (TOOMBS; WATERS, 2003). A TC ou RM, por serem exames de imagem de seç̧ão transversais são superiores para detectar a DDIV por seu melhor contraste e por reduzir as sobreposições em comparação com RX (HECHT et al., 2009).

A mielografia já foi 0 exame de escolha para fins diagnósticos, com 72 a $97 \%$ de sensibilidade de localização da lesão e em 89 a 100\% indicando 0 lado da compressão (BRISSON, 2010). Porém, podem ocorrer falhas no exame decorrente de hemorragia ou edema e múltiplas lesões graves ao paciente, por conta da contusão/compressão da ME, 0 que dificultará 0 aparecimento do contraste (ARIAS et al., 2007). Os efeitos colaterais mais observados são as convulsões em até 24 horas após realização do exame. Alguns pacientes apresentam aumento transitório da sintomatologia, que desaparece quando o contraste é reabsorvido. Em casos muito raros, 0 animal pode vir a óbito POR reação alérgica grave ao contraste utilizado ou por complicações advindas da anestesia (BRISSON, 2010).

A TC é o exame de imagem capaz de revelar a integridade dos DIV, podendo assim, diagnosticar alteração numa fase inicial (JENSEN; CHRISTENSEN, 2000). De acordo com Da Costa e Dewey (2017), na hérnia de disco Hansen tipo I, visualiza-se material hiperdenso dentro do canal vertebral, ME alterada em sua forma e diminuição da gordura epidural. Rosenblatt et al. (2018) demonstraram, em seu estudo com Dachshund, que entre as modalidades de RX, RM e TC, a última demonstrou maior acurácia para calcificação de disco (38,8\% dos escores positivos para calcificação). Stigen et al. (2019) corroboram com Rosenblatt et al. (2018) e afirmam que a alta sensibilidade da TC, torna esta modalidade altamente recomendada para Dachshund com sinais clínicos específicos de DDIV.

De acordo com Brisson (2010), a RM é considerada o melhor método diagnóstico para DDIV, 
sendo que ela é mais precisa para determinar o local e o lado da lesão. Como na RM, o tamanho da lesão tende a ser subestimando, é preciso atentar-se no momento da cirurgia para realização de uma janela superior do que a indicada no exame.

A RM consegue visualizar detalhes anatômicos com maior qualidade do que os exames citados anteriormente (DA COSTA; DEWEY, 2017). A RM consegue detectar alterações bioquímicas no DIV, como perda de água e proteoglicanos e diminuição da proporção de sulfato condroitina-queratina no NP, porém 0 exame não consegue diferenciar o disco calcificado de degenerado (BERGKNUT et al., 2011).

0 DIV degenerado no exame de imagem não serve como diagnóstico confirmatório de hérnia de disco, podendo este DIV extrudir ou não, pois nem todo material extrudido é mineralizado (WIDMER; THRALL, 2002).

0 diagnóstico definitivo da doença acontece durante o procedimento cirúrgico de descompressão espinhal (TOOMBS; WATERS, 2007), em que na extrusão é visto conteúdo discal no canal vertebral e na protrusão a projeção do AF (SHARP; WHEELER, 2005).

\subsection{TRATAMENTO}

Segundo Brisson (2010), o tratamento de cães com DDIV pode ser clínico ou cirúrgico, dependendo do grau de disfunção neurológica observadas.

0 tratamento clínico é realizado quando 0 animal possui dor ou se o déficit neurológico é mínimo (KRANENBURG et al., 2013; LECOUTEUR; GRANDY, 2004), desde que 0 paciente deambule e que não tenha apresentado sinais clínicos prévios de DDIV (LECOUTEUR; GRANDY, 2004), condizente com graus I ou || de graduação da doença (ARIAS et al., 2007). 0 protocolo baseia-se no uso de analgésicos e antiinflamatórios associados ao confinamento restrito em gaiola por quatro a seis semanas (SHARP; WHEELER, 2005; FESTUGATTO et al., 2008). 0 repouso auxilia na recuperação da inflamação da ME e facilita a reparação do DIV para não ocorrer extrusão adicional no local (LECOUTEUR; GRANDY, 2004). Segundo Da Costa e Dewey (2017), o uso de prednisona na dose de $0,5 \mathrm{mg} / \mathrm{kg}$ a cada 24 horas durante 15 dias, seguindo de $0,5 \mathrm{mg} / \mathrm{kg}$ a cada 48 horas durante mais 15 dias é recomendado nesses casos. Os corticoides são utilizados para redução de edema vasogênico, redução da compressão medular, diminuição da dor como consequência da melhora da inflamação das raízes nervosas e da isquemia (ARIAS et al., 2007).

$A$ associação da fisioterapia ao tratamento clínico de DDIV pode proporcionar uma recuperação mais rápida e adequada ao paciente. 0 protocolo fisioterapêutico deve ser individualizado, de acordo com local de lesão e viabilidade financeira do tutor do animal (FOSSUM, 2005). Como aparelhos de escolha utilizados na recuperação do paciente, 0 ultrassom possibilita analgesia, melhora do suprimento sanguíneo e cicatrização do tecido. Já a laserterapia atua diretamente na inflamação, com analgesia e redução dos níveis de serotonina, sendo assim realiza a diminuição das enzimas que induzem a dor no local da lesão (PRYOR; MILLIS, 2015).

A acupuntura também é uma boa opção para protocolo fisioterapêutico, pela analgesia que acontece na inserção das agulhas ao paciente. Estudos realizados em pacientes neurológicos demonstraram que alguns pontos de acupuntura podem regular fatores próinflamatórios, como interleucinas, ciclo-oxigenase 2 (COX-2), melhora da circulação local e redução dos efeitos de radicais livres na lesão (ROYNARD et al., 2017).

0 tratamento cirúrgico é indicado para animais com grau de déficit neurológico maior que II, em cães que não tenham tido sucesso ao tratamento clínico ou então recidiva da DDIV (SHARP; WHEELER, 2005).

A escolha da técnica cirúrgica varia de acordo com o local da lesão e posição, tendo como objetivo a descompressão espinhal. Na DDIV cervical a fenda ventral é comumente realizada, já na DDIV toracolombar a hemilaminectomia, mini-hemilaminectomia ou pediculectomia associadas à fenestração do DIV são mais utilizadas (BRISSON, 2010). 


\subsection{PROGNÓSTICO}

0 prognóstico é influenciado por diferentes fatores, especialmente pela presença ou ausência da dor profunda. A percepção da dor profunda é considerada inalterada quando a partir de um estímulo nocivo existe a percepção da dor (JEFFERY et al., 2016). Cães que apresentam dor profunda, após cirurgia descompressiva, possuem altas taxas de recuperação, de 86-96\% (BUSH et al., 2007) comparando com a taxa de recuperação de $50 \%$ dos cães que perderam a dor profunda (AIKAWA et al., 2012). Segundo Witsberger et al. (2012), nenhuma das avaliações isoladas fornecem precisão do prognóstico superior à de testes para a percepção da dor profunda. Medição de várias concentrações de biomarcadores em soro ou líquor é uma promessa considerável para 0 prognóstico futuro.

Considera-se como bom prognóstico os cães com DDIV cervical que apresentem sinais neurológicos leves e que mantém deambulação ambulatorial. 0 paciente que mantém a dor profunda antes da realização do procedimento cirúrgico possui 1,7 vezes mais chances de voltar a deambular normalmente (BRISSON, 2010).

Contrário à revisão de Brisson (2010), Jeffery et al. (2016) não relataram nenhuma evidência para um pior prognóstico para os cães que tiveram ausência de percepção de dor profunda por período superior a 48 horas após 0 início de paraplegia, os dados sugerem que os cães com paraplegia e perda da sensação de dor profunda não necessariamente necessitam de intervenção cirúrgica imediata.

\subsection{RECIDIVAS}

Podem ocorrer recidivas pelo mesmo DIV ou por acometimento em outros locais. Quando ocorrer uma recidiva, os sinais clínicos tendem a ser de maior gravidade, devendo-se avaliar 0 animal novamente e dependendo do seu grau de disfunção, optar por tratamento de escolha adequado à clínica observada. Normalmente, quando os sinais clínicos são mais graves, o veterinário deve optar por tratamento cirúrgico descompressivo (ARIAS et al., 2007).

De acordo com Aikawa et al. (2012), até 44\% dos cães apresentam recidiva, e destes de 6,4-12,7\% necessitam de nova intervenção cirúrgica. Observase também que até $25 \%$ dos cães condrodistróficos podem ficar com déficits neurológicos após a cirurgia, incluindo automutilação e incontinência fecal e/ou urinária.

\section{CONCLUSÃO}

É alta a incidência de DDIV na clínica de pequenos animais. A enfermidade pode acometer cães e gatos de todas as raças e idades, porém como vimos, os cães de raças condrodistróficas com maior predisposição e entre estes 0 Dachshund com condição superior, devido à sua conformação anatômica e pela carga genética do cromossomo $12 \mathrm{e}$ 18. Esta patologia é uma enfermidade comum, mesmo assim, a casuística permanece apresentando muitos desafios para 0 médico veterinário. Para um preciso diagnóstico é de suma importância a realização de um exame neurológico detalhado, 0 que propicia a identificação do local de lesão e assim, a correta realização de exames de imagem. Tais exames com a crescente evolução tecnológica proporcionaram significativas melhorias que promovem maior $\mathrm{e}$ melhor precisão diagnóstica e, consequentemente, alternativas e melhorias para o plano de tratamento de cada paciente. 


\section{REFERÊNCIAS}

AlKAWA, T. et al. Recurrent thoracolumbar intervertebral disc extrusion after hemilaminectomy and concomitant prophylactic fenestration in 662 chondrodystrophic dogs. Veterinary Surgery, v. 41, n. 3, p. 381-390, mar., 2012. Disponível em: https:// onlinelibrary.wiley.com/DOI/pdf/10.1111/j.1532950X.2012.00970.x. Acesso em: 18 Mar. 2019.

ARIAS, M. V. B. et al. Avaliação dos resultados clínicos após cirurgia descompressiva em cães com doença de disco intervertebral. Arquivo Brasileiro de Medicina Veterinária e Zootecnia. v. 59, n. 6, p. 1445-1450, 2007. Disponível em: http://www.scielo.br/scielo.php?script=sci_ arttext\&pid=S0102-09352007000600015. Acesso em: 28 Jan. 2019.

BERGKNUT, N. et al. The dog as an animal model for intervertebral disc degeneration?. Spine, $v$. 37, n. 5, p. 351-358, 2011. Disponível em: https:// journals.Iww.com/spinejournal/Abstract/2012/03010/ The_Dog_as_an_Animal_Model_for_Intervertebral_ Disc.2.aspx. Acesso em: 22 Fev. 2019.

\section{BERGKNUT, N. et al. Intervertebral disc}

\section{degeneration in the dog. Part 1: Anatomy}

and physiology of the intervertebral disc and characteristics of intervertebral disc degeneration. The Veterinary Journal, v. 195, n. 3, p. 282-291, 2013. Disponível em: https://www.sciencedirect.com/ science/article/pii/S1090023312004595. Acesso em: 15 Mar. 2019.

BRISSON, B. A. Intervertebral disc disease in dogs. Veterinary Clinics of North America: Small Animal Practice, Guelph, v. 40, p. 829-858, 2010. Disponível em: https://europepmc.org/abstract/med/20732594. Acesso em: 18 Jun. 2019.

BUSH, W. W. et al. Functional outcome following hemilaminectomy without methylprednisolone sodium succinate for acute thoracolumbar disk disease in 51 nonambulatory dogs. Journal of veterinary emergency and critical care, v. 17, n. 1, p. 72-76, 2007. Disponível em: https://onlinelibrary.wiley.com/ doi/pdf/10.1111/j.1476-4431.2006.00202.x. Acesso em: 08 Jun. 2019.

DA COSTA, R. C.; DEWEY, C. W. Neurologia Canina e Felina. $1^{a}$ ed. São Paulo: Editora Guará, 2017.

DE DECKER, S.; FENN, J. Acute herniation of nondegenerate nucleus Pulposus: acute noncompressive nucleus Pulposus extrusion and compressive hydrated nucleus Pulposus extrusion.

Veterinary Clinics of North America: Small Animal Practice, 2018. Disponível em: https://www.vetsmall. theclinics.com/article/S0195-5616(17)30093-1/ abstract. Acesso em: 17 Mai 2019.

DE RISIO, L. et al. Association of clinical and magnetic resonance imaging findings with outcome in dogs with presumptive acute noncompressive nucleus pulposus extrusion: 42 cases (2000-2007). Journal of the American Veterinary Medical Association, v. 234, n. 4, p. 495-504, 2009. Disponível em: https://avmajournals.avma.org/D0l/abs/10.2460/ javma.234.4.495. Acesso em: 12 Mai. 2019.

DENNY, H. R.; BUTTERWORTH, S. J. Cirurgia ortopédica em cães e gatos. 4. ed., São Paulo: Editora Roca, p. 193-205, 2006.

DOLERA, M. et al. Hydrated nucleus pulposus extrusion in dogs: correlation of magnetic resonance imaging and microsurgical findings. Acta Veterinaria Scandinavica, v. 57, n. 1, p. 58, 2015. Disponível em: https://actavetscand.biomedcentral.com/ articles/10.1186/s13028-015-0151-x. Acesso em: 05 Jan. 2019.

DORN, M.; SEATH, I. J. Neuter status as a risk factor for canine intervertebral disc herniation (IVDH) in dachshunds: a retrospective cohort study. Canine genetics and epidemiology, v. 5, n. 1, p. 11, 2018. Disponível em: https://cgejournal.biomedcentral.com/ articles/10.1186/s40575-018-0067-7. Acesso em: 20 Fev. 2019.

FENN, J. et al. Comparison of clinical signs and outcomes between dogs with presumptive ischemic myelopathy and dogs with acute noncompressive 
nucleus pulposus extrusion. Journal of the American Veterinary Medical Association, v. 249, n. 7, p. 767-775, 2016. Disponível em: https://avmajournals. avma.org/DOl/abs/10.2460/javma.249.7.767. Acesso em: 14 Abr. 2019.

\section{FERNÁNDEZ, V. L.; BERNARDINI, M. Neurologia em} cães e gatos. São Paulo: MEDVET livros, 2010. 452p.

FESTUGATTO, R. et al. Functional recovery of dogs with thoracolumbar intervertebral disk disease submitted the surgical treatment. Ciência Rural, vol. 38, n. 8, p. 2232-2238, nov., 2008. Disponível em: http://www.scielo.br/scielo.php?pid=S0103$84782008000800022 \&$ script=sci_arttext. Acesso em: 05 Mar. 2019.

FOSSUM, T. W. Small animal surgery. 3rd. St. Louis, M0: Mosby Elsevier, P it, 2005, 1610p.

HAKOZAKI, T. et al. Cervical intervertebral disk herniation in chondrodystrophoid and nonchondrodystrophoid small-breed dogs: 187 cases (1993-2013). Journal of the American Veterinary Medical Association, v. 247, n. 12, p. 1408-1411, 2015. Disponível em: https://avmajournals.avma.org/ D0l/abs/10.2460/javma.247.12.1408. Acesso em: 07 Fev. 2019.

HECHT, S. et al. Myelography vs. computed tomography in the evaluation of acute thoracolumbar intervertebral disk extrusion in chondrodystrophic dogs. Veterinary radiology \& ultrasound, v. 50, n. 4, p. 353-359, 2009. Disponível em: https://onlinelibrary.wiley.com/D0l/abs/10.1111/ j.1740-8261.2009.01549.x. Acesso em: 11 Mar. 2019.

HENKE, D. et al. Magnetic resonance imaging findings in dogs with traumatic intervertebral disk extrusion with or without spinal cord compression: 31 cases (2006-2010). Journal of the American Veterinary Medical Association, v. 242, n. 2, p. 217-222, 2013. Disponível em: https://avmajournals.avma.org/DOl/ abs/10.2460/javma.242.2.217. Acesso em: 25 Mai. 2019.
JEFFERY, N. D. et al. Factors associated with recovery from paraplegia in dogs with loss of pain perception in the pelvic limbs following intervertebral disk herniation. Journal of the American Veterinary Medical Association, v. 248, n. 4, p. 386-394, 2016. Disponível em: https://www.ncbi.nlm.nih.gov/ pubmed/26829270. Acesso em: 05 Jun. 2019.

JENSEN, V. F.; ARNBJERG, J. Development of intervertebral disk calcification in the dachshund: a prospective longitudinal radiographic study. Journal of the American Animal Hospital Association, v. 37, n. 3, p. 274-282, 2001. Disponível em: https:// www.jaaha.org/D0l/abs/10.5326/15473317-37-3274. Acesso em: 27 Jan. 2019.

JENSEN, V. F. et al. Quantification of the association between intervertebral disk calcification and disk herniation in Dachshunds. Journal of the American Veterinary Medical Association, v. 233, n. 7, p. 1090-1095, 2008. Disponível em: https://avmajournals.avma.org/DOl/abs/10.2460/ javma.233.7.1090. Acesso em: 09 Mai. 2019.

JENSEN, V. F.; CHRISTENSEN, K. A. Inheritance of disc calcification in the dachshund. Journal of Veterinary Medicine Series A, v. 47, n. 6, p. 331-340, 2000. Disponível em: https://onlinelibrary.wiley.com/DOI/ abs/10.1046/j.1439-0442.2000.00297.x. Acesso em: 02 Mai. 2019.

KRANENBURG, H. C. et al. Intervertebral disc disease in dogs-Part 2: Comparison of clinical, magnetic resonance imaging, and histological findings in 74 surgically treated dogs. The Veterinary Journal, v. 195, n. 2, p. 164-171, 2013. Disponível em: https://www.sciencedirect.com/science/article/pii/ S109002331200233X. Acesso em: 23 Mar. 2019.

LAPPALAINEN, A. K.; MÄKI, K.; LAITINEN-VAPAAVUORI, 0 . Estimate of heritability and genetic trend of intervertebral disc calcification in Dachshunds in Finland. Acta Veterinaria Scandinavica, v. 57, n. 1, p. 78, 2015. Disponível em: https://actavetscand. biomedcentral.com/articles/10.1186/s13028-0150170-7. Acesso em: 28 Jan. 2019. 
LECOUTEUR, R. A.; GRANDY, J. L. Doenças da medula espinhal. In: ETTINGER, S. J.; FELDMAN, E. C. Tratado de medicina interna veterinária: moléstias do cão e do gato. 5. ed. Rio de Janeiro: Guanabara Koogan, 2004, p. 664-694.

MANUNTA, M. L. et al. Hydrated nucleus pulposus herniation in seven dogs. The Veterinary Journal, v. 203, n. 3, p. 342-344, 2015. Disponível em: https://www.sciencedirect.com/science/article/pii/ S1090023314005255. Acesso em: 18 Fev. 2019.

MCKEE, M. Intervertebral disc disease in the dog 1. Pathophysiology and diagnosis. Practice, v. 22, n. 7 , p. 355, 2000. Disponível em: https://inpractice.bmj. com/content/22/7/355.short. Acesso em: 26 Fev. 2019.

MOGENSEN, M. S. et al. Validation of genomewide intervertebral disk calcification associations in dachshund and further investigation of the chromosome 12 susceptibility locus. Frontiers in genetics, v. 3, p. 225, 2012. Disponível em: https://www.frontiersin.org/articles/10.3389/ fgene.2012.00225/full. Acesso em: 06 Jun. 2019.

OLBY, N. The pathogenesis and treatment of acute spinal cord injuries in dogs. Veterinary Clinics: Small Animal Practice, v. 40, n. 5, p. 791-807, 2010. Disponível em: https://www.vetsmall.theclinics. com/article/S0195-5616(10)00065-3/abstract. Acesso em: 18 Abr. 2019.

OLBY, N.J.; JEFFERY, N.D. Pathogenesis and physiology of central nervous system disease and injury. In: TOBIAS, K.M.; JOHNSTON, S.A. Veterinary surgery small animal. 7. ed. St. Louis: Elsevier Saunders, 2012. p. 374-387.

OLBY, N. J. et al. A placebocontrolled, prospective, randomized clinical trial of polyethylene glycol and methylprednisolone sodium succinate in dogs with intervertebral disk herniation. Journal of veterinary internal medicine, v. 30, n. 1, p. 206-214, 2016. Disponível em: https://onlinelibrary.wiley.com/DOI/ full/10.1111/jvim.13657. Acesso em: 21 Mar. 2019.
PACKER, R.M.A. et al. Dachs Life 2015: an investigation of lifestyle associations with the risk of intervertebral disc disease in Dachshunds. Canine Genetics and Epidemiology, Hertfordshire, v. 3, n. 8, p. 1-15, 2016. Disponível em: https://cgejournal. biomedcentral.com/articles/10.1186/s40575-0160039-8. Acesso em: 31 Jan. 2019.

PARKER, H. G. et al. An expressed fgf 4 retrogene is associated with breed-defining chondrodysplasia in domestic dogs. Science, v. 325, n. 5943, 2009. Disponível em: https://science.sciencemag.org/ content/325/5943/995. Acesso em: 24 Jan. 2019.

\section{PENHA, E. M. et al. Hemivértebras com fusão} vertebral em cão - Relato de caso. PUBVET, v. 4, p. Art. 850-857, 2010. Disponível em: http://www. pubvet.com.br/artigo/2307/hemiveacutertebrascom-fusatildeo-vertebral-em-catildeo-ndashrelato-de-caso. Acesso em: 11 Mar. 2019.

PRYOR, B.; MILLIS, D. L. Therapeutic laser in veterinary medicine. Veterinary Clinics: Small Animal Practice, v. 45, n. 1, p. 45-56, 2015. Disponível em: https://www.vetsmall.theclinics.com/ article/S0195-5616(14)00135-1/abstract. Acesso em: 28 Mar. 2019.

ROHDIN, C. et al. Prevalence of radiographic detectable intervertebral disc calcifications in Dachshunds surgically treated for disc extrusion. Acta Veterinaria Scandinavica, v. 52, n. 1, p. 24, 2010. Disponível em: https://actavetscand.biomedcentral. com/articles/10.1186/1751-0147-52-24. Acesso em: 13 Abr. 2019.

ROSENBLATT, A. J. et al. Scorer and modality agreement for the detection of intervertebral disc calcification in Dachshunds. Acta Veterinaria Scandinavica, v. 60, n. 1, p. 60-62, 2018. Disponível em: https://actavetscand.biomedcentral.com/ articles/10.1186/s13028-018-0416-2. Acesso em: 22 Jan. 2019.

ROWLAND, J. W. et al. Current status of acute spinal cord injury pathophysiology and emerging therapies: promise on the horizon. Neurosurgical 
focus, v. 25, n. 5, p. E2, 2008. Disponível em: https://thejns.org/focus/view/journals/neurosurgfocus/25/5/article-pE2.xml. Acesso em: 08 Fev. 2019.

ROYAUX, E. et al. Detection of compressive hydrated nucleus pulposus extrusion in dogs with multislice computed tomography. The Veterinary Journal, v. 216, p. 202-206, 2016. Disponível em: https:// www.sciencedirect.com/science/article/pii/ S1090023316301277. Acesso em: 19 Mar. 2019.

ROYNARD, P et al. Acupuncture for Small Animal Neurologic Disorders. Veterinary Clinics: Small Animal Practice, v. 48, n. 1, p. 201-219, 2017. Disponível em: https://www.vetsmall.theclinics.com/ article/S0195-5616(17)30092-X/abstract. Acesso em: 10 Mar. 2019.

SHARP, N. J. H.; WHEELER, S. J. Small animal spinal disorders: diagnosis and surgery. 2.ed. Philadelphia: Elsevier Mosby, 2005. [363p.]. Disponível em: https://www.cabdirect.org/cabdirect/ abstract/19942214873. Acesso em: 12 Abr. 2019.

SMOLDERS, L. A. et al. Intervertebral disc degeneration in the dog. Part 2: chondrodystrophic and non-chondrodystrophic breeds. The veterinary journal, v. 195, n. 3, p. 292-299, 2013. Disponível em: https://www.sciencedirect.com/science/article/ pii/S1090023312004339. Acesso em: 25 Abr. 2019.

STIGEN, Ø.; CIASCA, T.; KOLBJØRNSEN, $\emptyset$.

Calcification of extruded intervertebral discs in dachshunds: a radiographic, computed tomographic and histopathological study of 25 cases. Acta Veterinaria Scandinavica, v. 61, n. 1, p. 13, 2019. Disponível em: https://actavetscand.biomedcentral. com/articles/10.1186/s13028-019-0448-2. Acesso em: 09 Jun. 2019.

TOOMBS J. P.; WATERS, D. J. Intervertebral disc disease. In: SLATTER, Douglas H. (Ed.) Textbook of Small Animal Surgery. vol. 1. 3th ed. Saunders Elsevier, Philadelphia. 2003, p.1193-1208.

TOOMBS, J. P.; WATERS, D. J. Afecção do disco intervertebral. In: SLATTER, Douglas. Manual de cirurgia de pequenos animais. 3. ed. São Paulo: Manole, 2007. p. 1193-1208.

WIDMER, W. R.; THRALL, D. E. Canine and feline intervertebral disc disease, myelography, and spinal cord disease. In: THRALL, D.E. Textbook of veterinary diagnostic radiology. 4. ed. St. Louis: Elsevier Saunders, 2002. p. 110-127.

WITSBERGER, T. H. et al. As associações entre biomarcadores fluido cerebrospinal e evolução neurológica de longo prazo em cães com hérnia de disco intervertebral aguda. Journal of the American Veterinary Medical Association. v. 240, p. 555562, 2012. Disponível em: https://www.repository.utl. pt/handle/10400.5/12005. Acesso em: 17 Mar. 2019.

Recebido em: 02/09/2019

Aceito em: 14/10/2019 